\title{
EFFECTIVE PERMEABILITY OF FRACTURED POROUS MEDIA WITH FRACTAL DUAL-POROSITY MODEL
}

\author{
PENG XU, $,+,, \S, \|$ HAICHENG LIU, * AGUS PULUNG SASMITO, $,, \boldsymbol{\uparrow}, \|$ \\ SHUXIA QIU* and CUIHONG LI* \\ ${ }^{*}$ College of Science, China Jiliang University, Hangzhou 310018, P. R. China \\ †State Key Laboratory of Oil and Gas Reservoir Geology and Exploitation \\ Southwest Petroleum University, Chengdu 610500, P. R. China \\ ${ }^{\ddagger}$ Department of Mining and Materials Engineering \\ McGill University, Montreal, H3A 2A7 Canada \\ §xupeng@cjlu.edu.cn \\ 『agus.sasmito@mcgill.ca
}

Received February 13, 2017

Revised April 11, 2017

Accepted April 17, 2017

Published May 26, 2017

\begin{abstract}
As natural fractures show statistically fractal scaling laws, fractal geometry has been proposed and applied to model the fracture geometry and to study the hydraulic properties of fractured porous media. In this paper, a fractal dual-porosity model is developed to study the single-phase fluid flow through fractured porous media. An analytical expression for effective permeability of fractured porous media is derived, which depends on the fractal dimension and fracture aperture. The effect of fractal dimensions for fracture aperture distribution and tortuosity, the ratio of minimum to maximum fracture apertures and fracture fraction on the effective permeability have been discussed. In addition, a power law relationship between the effective
\end{abstract}

\footnotetext{
" Corresponding authors.

This is an Open Access article published by World Scientific Publishing Company. It is distributed under the terms of the Creative Commons Attribution 4.0 (CC-BY) License. Further distribution of this work is permitted, provided the original work is properly cited.
} 
permeability and fracture fraction is proposed to predict the equivalent hydraulic properties of fractured porous media. Compared with empirical formulas for effective permeability, the present fractal dual-porosity model can capture the statistical characteristics of fractures and shed light on the transport mechanism of fractured porous media.

Keywords: Fractured Porous Media; Dual-Porosity Model; Fractal; Effective Permeability.

\section{INTRODUCTION}

The equivalent hydraulic properties of fractured porous media are of vital importance to oil and gas reservoirs, mining, groundwater resources, gas storage, nuclear waste disposal, geothermal energy, etc! 14 However, due to the complexity and irregularity of fractured porous media, it is a challenge to explicitly model the discrete fractures and to specify the equivalent hydraulic properties of fractured porous media. ${ }^{56}$ The effective permeability of fractured porous media depends on both fracture geometry (e.g. fracture length and aperture distribution, surface roughness, dead-end, intersections, etc.) and surrounding conditions (e.g. hydraulic gradient, stress, anisotropy, etc.). Various continuum models, discrete fracture models and dual-porosity models have been proposed to study the fluid flow and estimate the effective permeability of fractured porous media. ${ }^{7}[10$

The concept of dual porosity (dual domain) can be used to model the fluid flow on various scales in fractured porous media ${ }^{1112}$ In the dual-porosity model, a fractured porous medium is composed of fracture system and porous matrix. The embedded fractures in porous matrix take dominated effect on the transport process. However, it is difficult to explicitly model the geometry of discrete fractures in a dual-porosity media as the dimension of fractures can vary over several orders of magnitude. As natural fractures have been shown to exhibit statistically fractal characteristics, fractal geometry has been proposed to model the geometrical and topological structure of the fracture system and to study the transport properties of fractured porous media. $13-15$ Chang and Yortsos $\frac{16}{16}$ proposed a fractal model for the radial permeability of fractured reservoirs, in which the relation between transport exponent and fractal dimension is unknown. $\mathrm{Xu}$ et al. ${ }^{17] 18}$ employed the fractal tree-like networks to model the fracture system and developed a fractal dual-domain model for fractured porous media, and the effective planar linear and radial permeabilities have been proposed. They also carried out MonteCarlo simulation on the radial flow in fractured porous media based on the fractal capillary bundle model and indicated that the effective radial permeability relates to the geometrical parameters of well and size distribution of fractures ${ }^{19}$ According to the fractal dual-domain model by Xu et al., the starting pressure gradient for Bingham fluids, capillary pressure effect, relative permeability, gas permeability, gas diffusion coefficient and transient flow of fractured porous media have also been discussed $20 \mid 23$ Jafari and Babadagli24-26 found that the equivalent fracture network permeability is determined by the fractal properties of fracture networks; they presented a permeability expression with multiple regression analysis of random fractures by the fractal geometry and percolation theory. Liu et al.27 calculated the effective permeability of randomly distributed fracture networks by fractal scaling law and Monte-Carlo method and discussed the effect of fractal dimensions of geometric distribution and tortuosity on the hydraulic behavior of fracture networks. Miao et al. ${ }^{28}$ presented a fractal model for the permeability of fractured rocks, in which the fracture length distribution, fracture fraction and fracture azimuth as well as fracture dip angle have been taken into account.

Although many theoretical and numerical investigations by fractal geometry have been performed to predict the equivalent hydraulic properties of fractured porous media, the influence mechanisms of geometrical and flow parameters on the effective permeability are still not well understood. Additionally, many parameters in existing fractal models for effective permeability of fractured porous media are difficult to measure in practical applications. There is a strong demand for a direct relationship between permeability and fracture fraction (porosity), in order to estimate the equivalent hydraulic properties of fractured porous media. Therefore, a fractal dual-porosity model is developed for fractured porous media according to the fractal scaling laws of fractures, and the effective permeability is derived accordingly. In addition, the relationship between permeability and geometrical structures is also discussed. 


\section{FRACTAL DUAL-POROSITY MODEL}

A fractal dual-porosity model for laminar flow of incompressible Newtonian fluid through a fractured porous medium is presented in this part. A representative volume element (REV) with length $L$, width $W$ and height $H$ is illustrated in Fig. 1. Based on the dual-porosity theory, the fractures with fractal scaling law were parallelly embedded into a homogeneous porous matrix with low permeability $\left(K_{\mathrm{m}}\right)$, and the height of the fractures and porous matrix was taken as the same $(h=H)$. The fracture aperture and length were assumed to follow fractal scaling laws. The fractal probability density function for the fracture aperture on the cross-sectional area in fractured porous media can be expressed as 29 .

$$
f(w)=D_{w} w_{\min }^{D_{w}} w^{-\left(D_{w}+1\right)},
$$

where $w$ is the fracture aperture and $w_{\min }$ and $w_{\max }$ denote the minimum and maximum fracture apertures, respectively. The fractal dimension for fracture aperture distribution is in the range of $1<D_{w}<2$ in two-dimensional space. The probability density function should satisfy the normalization condition, which gives $\left(w_{\min } / w_{\max }\right)^{D_{w}}=0$.

The tortuous fracture length $l$ can be calculated with the straight length $L$ and fracture aperture $w$ as well as tortuosity fractal dimension $D_{t}$, according to the following fractal scaling law 29 :

$$
l(w)=L^{D_{t}} w^{1-D_{t}} .
$$

The tortuosity fractal dimension $D_{t}$ corresponds to the tortuousness of fractures, the value of which is in the range of $1<D_{t}<2$ in two-dimensional space.

For the steady laminar flow through fractures, the flow rate along the flow direction through a fracture can be determined by the cubic law:

$$
q(w)=\frac{w^{3} h}{12 \mu} \frac{\Delta p}{l}
$$

where $\Delta p$ is the pressure drop across a fracture along the flow direction and $\mu$ is the fluid viscosity.

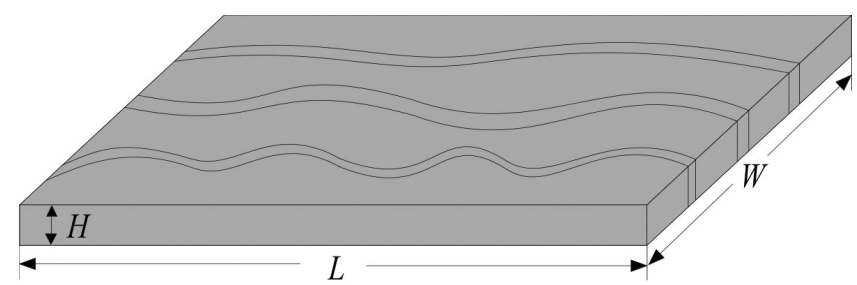

Fig. 1 A schematic graph of fractal dual-porosity model for fractured porous media.
The fracture aperture $w$ is generally much smaller than its length $l$ and height $h$. Then, the total flow rate through all the fractures with fractal scaling laws can be obtained by integrating Eq. (3) from the minimum fracture aperture to the maximum fracture aperture with the aid of Eqs. (1) and (2):

$$
\begin{aligned}
Q= & \int_{w_{\min }}^{w_{\max }} \frac{w^{3} h}{12 \mu} \frac{\Delta p}{L^{D_{t}} w^{1-D_{t}}} D_{w} w_{\max }^{D_{w}} w^{-\left(D_{w}+1\right)} d w \\
= & \frac{h w_{\max }^{2+D_{t}}}{12 \mu} \frac{D_{w}}{2+D_{t}-D_{w}} \frac{\Delta p}{L^{D_{t}}} \\
& \times\left[1-\left(\frac{w_{\min }}{w_{\max }}\right)^{2+D_{t}-D_{w}}\right] .
\end{aligned}
$$

Darcy's law for Newtonian fluid flow in porous media can be written as:

$$
Q=\frac{K}{\mu} A \frac{\Delta p}{L},
$$

where the cross-sectional area is $A=W H$ and $K$ is the fluid permeability. Combining Eqs. (4) and (5), the effective permeability for fractured porous media can be obtained as:

$$
\begin{aligned}
K= & \frac{1}{12} \frac{w_{\max }^{2+D_{t}}}{W L^{D_{t}-1}} \frac{D_{w}}{2+D_{t}-D_{w}} \\
& \times\left[1-\left(\frac{w_{\min }}{w_{\max }}\right)^{2+D_{t}-D_{w}}\right] .
\end{aligned}
$$

Based on the normalization condition of the fractal probability density function the ratio of minimum fracture aperture to the maximum fracture aperture is much less than 1.0, that is, $w_{\min } \ll w_{\max }$. Therefore, the effective permeability of the fractured porous media can be written as:

$$
K=\frac{L}{12 W} \frac{D_{w}}{2+D_{t}-D_{w}} \frac{w_{\max }^{2+D_{t}}}{L^{D_{t}}} .
$$

The dimensionless effective permeability can be expressed as:

$$
K^{+}=\frac{K}{w_{\max }^{3} /(12 W)}=\frac{D_{w}}{2+D_{t}-D_{w}} \frac{w_{\max }^{D_{t}-1}}{L^{D_{t}-1}} .
$$

According to the fractal geometry of the fractures, the total fracture volume can be calculated by the integration of an arbitrary fracture from minimum to maximum fracture size with the aid of Eqs. (10) and (2). In addition, the fracture fraction (porosity) can be obtained as the ratio of total fracture volume 
to the REV volume:

$$
\begin{aligned}
\phi= & \frac{w_{\max }^{2-D_{t}} L^{D_{t}}}{W L} \frac{D_{w}}{2-D_{t}-D_{w}} \\
& \times\left[1-\left(\frac{w_{\min }}{w_{\max }}\right)^{2-D_{t}-D_{w}}\right] .
\end{aligned}
$$

The matrix permeability was approximated to be a constant, and the effective permeability of fractured porous media can be calculated by the arithmetic mean of fracture permeability and porous matrix permeability ${ }^{3}$ As fractures behave as high permeability paths for fluid flow, the matrix permeability was neglected for simplification here. For the simple case of straight fractures $\left(D_{t}=1\right)$, the effective permeability and porosity are $K=D_{w} w_{\max }^{3} /[12 W(3-$ $\left.\left.D_{w}\right)\right]$ and $\phi=\left(w_{\max } / W\right)\left[\left(D_{w} /\left(1-D_{w}\right)\right][1-\right.$ $\left.\left(w_{\min } / w_{\max }\right)^{1-D_{w}}\right]$, respectively. Equations (6) -(8) indicate that the effective permeability of fractured porous media is related to the microstructures of fractures. Once the fractal scaling laws of fractures are determined, the effective permeability can be calculated accordingly. Also, the relationship between the effective permeability and fracture fraction can be addressed based on Eqs. (7) and (9).

\section{RESULTS AND DISCUSSION}

The values of the parameters used in this section are summarized as: $W=L=100 \mathrm{~m}, w_{\max }=0.01 \mathrm{~m}$ and $w_{\min }=0.002,0.001,0.0005$ and $0.0001 \mathrm{~m}$. Generally, the fractal dimensions for fracture aperture and tortuous length can be determined by the boxcounting method, which is based on the image

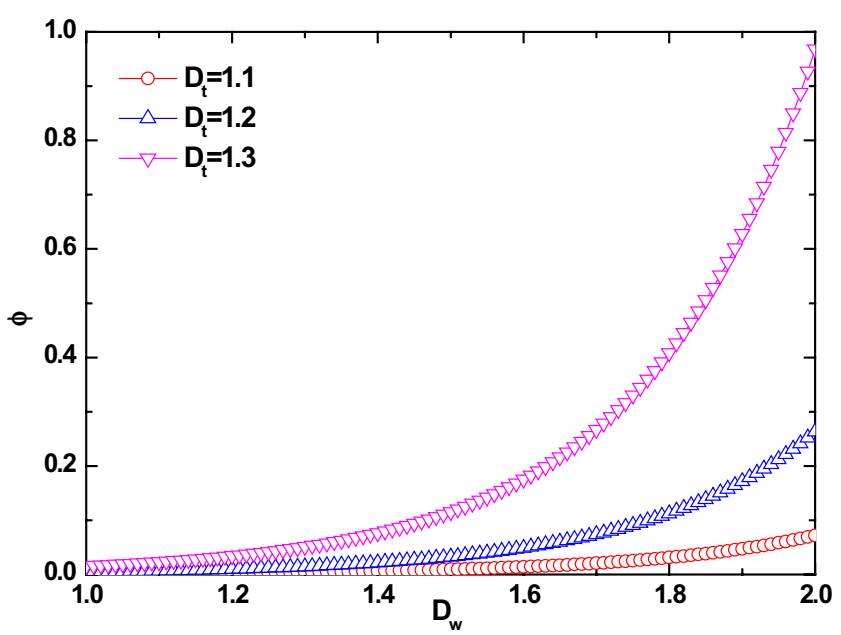

Fig. 2 The relationship between the fracture fraction and fractal dimensions at $w_{\min } / w_{\max }=0.01$. analysis of a unit cell ${ }^{[30}$ According to the physical meaning of the fractal dimension, larger fractal dimension means larger space occupied by fractures. Thus, it can be seen in Fig. 2 that fracture fraction increases as the fractal dimension for fracture aperture increases. The value of fracture fraction is close to 0 as the fractal dimension for fracture aperture is near 1.0. It is consistent with the practical situation because there is no fracture as the fractal dimension for fracture aperture distribution is 1.0. Also, increased tortuosity fractal dimension can clearly enhance fracture fraction, which can be attributed to the enhanced fracture length with increased tortuosity fractal dimension for fractures.

Figure 3 shows the relationship between the effective permeability and fracture fraction (porosity). The effective permeability of fractured porous media increases with increased fracture fraction.

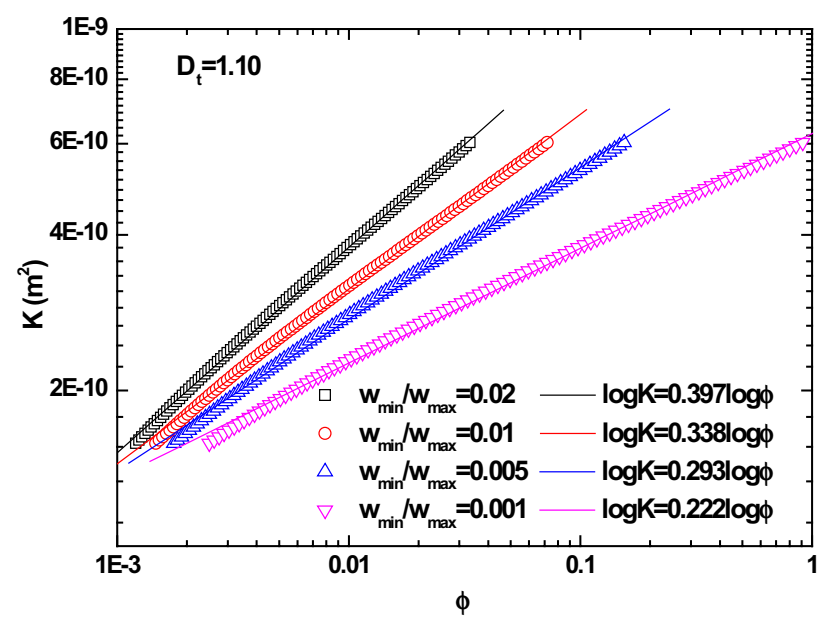

(a)

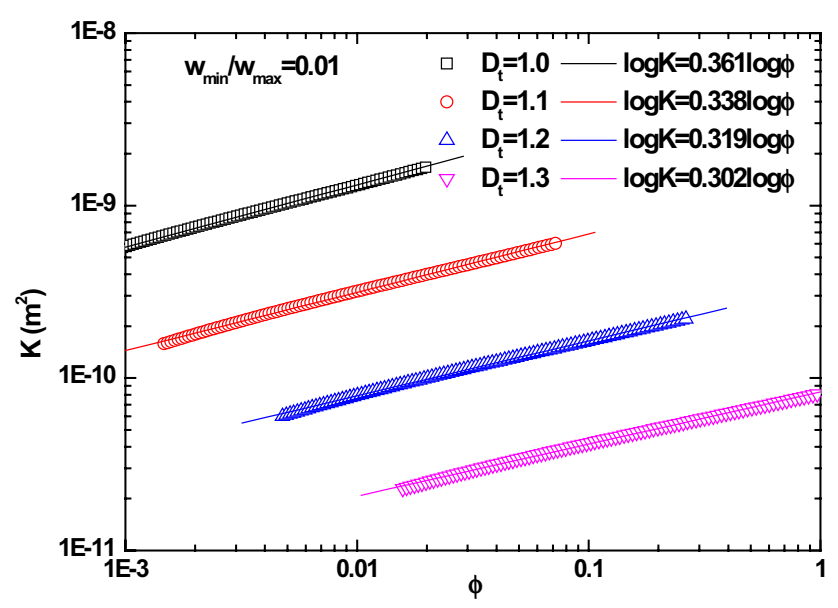

(b)

Fig. 3 The relationship between the effective permeability and fracture fraction. 
The value of effective permeability can be reduced by decreased ratio of minimum to maximum fracture aperture and increased tortuosity fractal dimension. It can be explained as that decreased ratio of minimum to maximum fracture aperture under fixed porosity and fractal dimension for fracture aperture corresponds to reduced fracture aperture. In addition, increased tortuosity fractal dimension meaning increased tortuousness of fractures can enhance flow resistance and lower effective permeability. A clear scaling law between the effective permeability and fracture fraction has been found as $K \sim \phi^{\alpha}$. The power law correlations between permeability and porosity have been proposed and proven by many researchers in different kinds of fractured porous media. ${ }^{5}$ Among the two common models for permeability-porosity relationship, power law scaling and exponential function, the former one is most widely accepted method due to its simplistic form and limited parameters. Many power law permeability-porosity models such as semi-empirical Kozeny-Carman model,, 31 modified Kozeny-Carman models for species porous medium,, $32 \sqrt{33}$ percolation theory,, 34 transient test,, 35 etc. have been proposed. However, the exponent parameter in the existing power law correlations depends on material properties and other structural parameters and should be determined by fitting experimental data. Further calculation indicates that the scaling factor between the effective permeability and fracture fraction depends on the fractal dimensions and ratio of minimum to maximum fracture aperture. The value of scaling factor is $\alpha=0.397,0.338,0.293$ and 0.222 for $w_{\min } / w_{\max }=$ $0.02,0.01,0.005$ and 0.001 under tortuosity fractal dimension $D_{t}=1.10$. It takes the value of $\alpha=0.361,0.338,0.319,0.302$ and 0.288 for the tortuosity fractal dimension of $D_{t}=1.0,1.1,1.2$ and 1.3 under $w_{\min } / w_{\max }=0.01$. With the analytical expressions for effective permeability and fracture fraction (Eqs. (7) and (9)), the scaling laws between the effective permeability and fracture fraction can be derived under different conditions.

Figure 4 illustrates the effect of fractal dimensions for fracture aperture distribution and tortuous fracture length on the dimensionless effective permeability of fractured porous media. It can be found in Fig. 4 that both fractal dimensions for fracture aperture distribution and tortuous fracture length show significant effect on the effective permeability of fractured porous media. The effective permeability of fractured porous media can

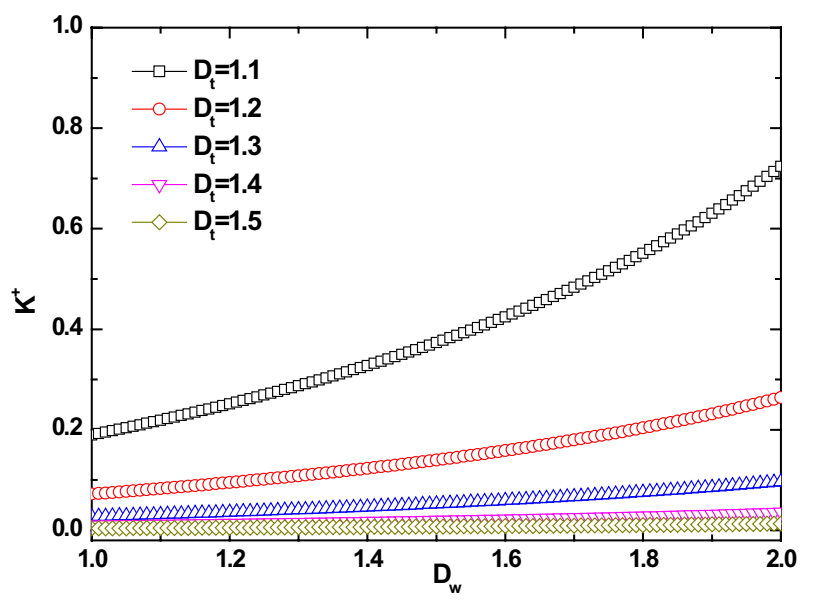

Fig. 4 The effect of fractal dimensions on the dimensionless effective permeability of fractured porous media.

be increased with the increment of fractal dimension for fracture aperture distribution $D_{w}$. This can be attributed to the increased porosity with the increase of aperture fractal dimension. However, the increase of tortuosity fractal dimension $D_{t}$ can drastically decrease the effective permeability. It can be seen from Fig. 4 that dimensionless effective permeability is very low when the tortuosity fractal dimension is large $\left(D_{t}=1.4\right.$ and 1.5$)$.

\section{CONCLUDING REMARKS}

A fractal dual-porosity model has been developed with the fractal scaling laws of fracture aperture distribution and tortuous fracture length for fractured porous media. The analytical expressions for effective permeability and fracture fraction have been derived, and the relationship between the effective permeability and fractal dimensions has been discussed. It has been found that the effective permeability of fractured porous media increases with the increase of fractal dimension for fracture aperture distribution and the decrease of tortuosity fractal dimension. A power law relationship $\left(K \sim \phi^{\alpha}\right)$ has been addressed for the effective permeability and fracture fraction, in which the exponent factor depends on the fractal dimension and ratio of minimum to maximum fracture aperture. The present fractal dual-porosity model may provide significant theoretical basis for geoscience and geoengineering as well as chemical engineering and so on. It should also be pointed out that the coupling between the porous matrix and the fracture system may be taken into account to improve the accuracy of the fractal dual-porosity model. 


\section{ACKNOWLEDGMENTS}

This work was jointly supported by the Open Fund (PLN1402) of State Key Laboratory of Oil and Gas Reservoir Geology and Exploitation (Southwest Petroleum University) and Zhejiang Provincial Natural Science Foundation of China through Grant numbers LY16A020002 and LQ16E060002.

\section{REFERENCES}

1. I. I. Bogdanov, V. V. Mourzenko, J. F. Thovert and P. M. Adler, Effective permeability of fractured porous media in steady state flow, Water Resour. Res. 39(1) (2003) 1023.

2. D. X. Zhang and Q. J. Kang, Pore scale simulation of solute transport in fractured porous media, Geophys. Res. Lett. 31(12) (2004) L12504.

3. M. J. Ronayen and S. M. Gorelick, Effective permeability of porous media containing branching channel networks, Phys. Rev. E 73(2) (2006) 026305.

4. M. Sahimi, Flow and Transport in Porous Media and Fractured Rock: From Classical Methods to Modern Approaches, 2nd edition (Wiley-VCH, 2011).

5. J. Ma, Review of permeability evolution model for fractured porous media, J. Rock Mech. Geotech. Eng. 7 (3) (2015) 351-357.

6. R. Liu, B. Li, Y. Jiang and N. Huang, Review: Mathematical expressions for estimating equivalent permeability of rock fracture networks, Hydrogeol. J. 24(7) (2016) 1623-1649.

7. Y. S. Wu, Numerical simulation of single-phase and multiphase non-Darcy flow in porous and fractured reservoirs, Transp. Porous Media 49(2) (2002) 209240.

8. H. Hoteit and A. Firoozabadi, An efficient numerical model for incompressible two-phase flow in fractured media, Adv. Water Resour. 31(6) (2008) 891-905.

9. Y. L. Zhao, L. H. Zhang et al., Analysis of horizontal well pressure behaviour in fractured low permeability reservoirs with consideration of the threshold pressure gradient, J. Geophys. Eng. 10(3) (2013) 035014 .

10. N. Huang, Y. Jiang, R. Liu and B. Li, A fast calculation method for estimating the representative elementary volume of three-dimensional fracture network, Special Top. Rev. Porous Media 7(2) (2016) 99-106.

11. G. I. Barenblatt, Iu. P. Zheltov and I. N. Kochina, Basic concepts in the theory of seepage of homogeneous liquids in fissured rocks, J. Appl. Math. Mech. 24(5) (1960) 1286-1303.

12. J. E. Warren and P. J. Root, The behavior of naturally fractured reservoirs, Soc. Petrol Engrs. J. 3(03) (1963) 245-255.
13. M. Sahimi, Flow phenomena in rocks: From continuum models to fractals, percolation, cellular automata, and simulated annealing, Rev. Mod. Phys. 65(4) (1993) 1393-1534.

14. E. Charkaluk M. Bigerelle and A. Iost, Fractals and fracture, Eng. Fract. Mech. 61(1) (1998) 119-139.

15. E. Bonnet, O. Bour, N. E. Odling et al., Scaling of fracture systems in geological media, Rev. Geophys. 39(3) (2001) 347-383.

16. J. Chang and Y. C. Yortsos, Pressure transient analysis of fractal reservoirs, SPE Form. Eval. 5(01) (1990) 31-38.

17. P. Xu, B. Yu, Y. Feng and Y. Liu, Analysis of permeability for the fractal-like tree network by parallel and series models, Phys. A 369(2) (2006) 884-894.

18. P. Xu, B. Yu, Y. Feng and M. Zou, Permeability of the fractal disk-shaped branched network with tortuosity effect, Phys. Fluids 18(7) (2006) 078103.

19. P. Xu, B. M. Yu, X. Qiao, S. Qiu and Z. Jiang, Radial permeability of fractured porous media by Monte Carlo simulations, Int. J. Heat Mass Transf. 57(1) (2013) 369-374.

20. Q. Zheng and B. M. Yu, A fractal permeability model for gas flow through dual-porosity media, J. Appl. Phys. 111(2) (2012) 024316.

21. Q. Zheng, J. Xu, B. Yang and B. Yu, Research on the effective gas diffusion coefficient in dry porous media embedded with a fractal-like tree network, Phys. A 392(6) (2013) 1557-1566.

22. X.-H. Tan, X.-P. Li, J.-Y. Liu, C. Tang and J.-M. Li, Pressure transient analysis of dual fractal reservoir, J. Appl. Math. 2013 (2013) 137518.

23. P. Xu, C. Li, S. Qiu and A. P. Sasmito, A fractal network model for fractured porous media, Fractals 24(2) (2016) 1650018.

24. A. Jafari and T. Babadagli, Effective fracture network permeability of geothermal reservoirs, Geothermics 40(1) (2011) 25-38.

25. A. Jafari and T. Babadagli, Estimation of equivalent fracture network permeability using fractal and statistical network properties, J. Petrol Sci. Eng. 9293 (2012) 110-123.

26. A. Jafari and T. Babadagli, Relationship between percolation-fractal properties and permeability of 2D fracture networks, Int. J. Rock Mech. Min. Sci. 60 (2013) 353-362.

27. R. C. Liu, Y. J. Jiang, B. Li and X. S. Wang, A fractal model for characterizing fluid flow in fractured rock masses based on randomly distributed rock fracture networks, Comp. Geotech. 65 (2015) 45-55.

28. T. J. Miao, B. M. Yu, Y. G. Duan and Q. T. Fang, A fractal analysis of permeability for fractured rocks, Int. J. Heat Mass Transf. 81 (2015) 75-80.

29. B. M. Yu and P. Cheng, A fractal permeability model for bi-dispersed porous media, Int. J. Heat Mass Transf. 45(14) (2002) 2983-2993. 
30. J. Feder and A. Aharony, Fractals in Physics (NorthHolland, Amsterdam, 1989).

31. P. Xu and B. Yu, Developing a new form of permeability and Kozeny-Carman constant for homogeneous porous media by means of fractal geometry, Adv. Water Resour. 31(1) (2008) 74-81.

32. A. Costa, Permeability-porosity relationship: A reexamination of the Kozeny-Carman equation based on a fractal pore-space geometry assumption, Geophys. Res. Lett. 33(2) (2006) L02318.

33. B. Xiao, X. Tu, W. Ren and Z. Wang, Modeling for hydraulic permeability and Kozeny-Carman constant of porous nanofibers using a fractal approach, Fractals 23(03) (2015) 1550029.

34. M. Sahimi, Application of Percolation Theory (Taylor and Francis, London, UK, 1994).

35. S. Ghabezloo, J. Sulem and J. Saint-Marc, Evaluation of a permeability-porosity relationship in a low-permeability creeping material using a single transient test, Int. J. Rock Mech. Min. Sci. 46(4) (2009) 761-768. 